Z Rheumatol 2016 $75: 32-40$

DOI 10.1007/s00393-015-0037-3

Online publiziert: 26. Januar 2016

(c) Springer-Verlag Berlin Heidelberg 2016

Redaktion

H.-P. Tony, Würzburg

CrossMark

\author{
H. Burkhardt ${ }^{1} \cdot$ P. Weisenseel ${ }^{2}$ M. A. Radtke ${ }^{3} \cdot K_{\text {. Krüger }}^{4}$ \\ 'Abteilung Rheumatologie \& Fraunhofer IME, Projektgruppe Translationale Medizin und Pharmakologie \\ Universitätsklinikum Frankfurt, Medizinische Klinik II, Goethe Universität Frankfurt am Main, Frankfurt \\ am Main, Deutschland \\ ${ }^{2}$ Dermatologikum Hamburg, Hamburg, Deutschland \\ ${ }^{3}$ Institut für Versorgungsforschung in der Dermatologie, Universitätsklinikum Eppendorf, Hamburg, \\ Deutschland \\ ${ }^{4}$ Rheumatologisches Praxiszentrum St. Bonifatius, München, Deutschland
}

\title{
Hautmalignome und immunmodulierende Antirheumatikatherapie
}

TD wird ohne weitere Risikofaktoren keine weitere Diagnostik empfohlen. Erst ab 1,0 mm TD werden die Exstirpation des Sentinellymphknotens, Bildgebung und die Bestimmung von Tumormarkern im Serum empfohlen. Die Nachsorge erfolgt stadienabhängig alle 3 bzw. 6 Monate.

In Deutschland sind im Jahr 2008 knapp 18.000 Melanome neu diagnostiziert worden. Die Inzidenz in Deutschland beträgt ca. 20/100.000 und in Australien 50-60/100.000 Einwohner. Das Hauptmanifestationsalter liegt zwischen dem 45. und 60. Lebensjahr, und das Risiko, im Leben ein Melanom zu entwickeln, beträgt in Deutschland in etwa 1:200, in Australien 1:50. Risikofaktoren sind eine starke UV-Belastung mit wiederkehrenden Sonnenbrandschädigungen der Haut sowie erbliche Prädisposition. Die Aktivierung des Immunsystems ist ein Schlüsselfaktor in der Tumorabwehr des malignen Melanoms, sodass Krankheitszustände mit kompromittierter Immunkompetenz, z. B. die dauerhafte Immunsuppression nach Organtransplantation, Risikofaktoren der Kanzerogenese sind [1]. Analog stellt sich daher die Frage, inwiefern die RA per se bzw. ihreimmunmodulierende pharmakotherapeutische Behandlung mit synthetischen oder biologischen DMARDs über eine Kompromittierung der Tumorimmunität das Risiko für die Melanomentstehung erhöht.
Unter den NMSC dominieren das BCC und SCC, die auch als sog. „weißer Hautkrebs" bezeichnet werden. Sie gehören zu den häufigsten Neoplasien überhaupt und sind für etwa $40 \%$ aller Malignome in der Bevölkerung verantwortlich [2], wobei das BCC ca. 4-fach häufiger als das SCC vorkommt. Metastasierungen treten beim BCC fast nie und bei SCC sehr selten auf. Für beide Formen der NMSC mit einem Erkrankungsgipfel um das 70. Lebensjahr sind neben einem prädisponierenden hellen - Hauttyp chronische Exposition zu Kanzerogenen (z. B. Arsen) sowie die kumulative UV-Expositionsdosis weitere Risikofaktoren. Aktinische Keratosen, die noch weitaus häufiger als BCC und SCC vorkommen und an sonnenexponierten Arealen im höheren Lebensalter auftreten, sind potenzielle Vorstufen des SCC bzw. histologisch bereits initiale SCCs. Aktinische Keratosen können sich aber auch spontan wieder zurückbilden, und nur aus einem kleinen Teil der Läsionen entwickeln sich im Verlauf invasive SCCs. Eine chronische Immunsuppression nach Organtransplantation erhöht ebenfalls das Erkrankungsrisiko (für das BCC 10-fach und für das SCC 20- bis 100-fach [3]) und führt auch $\mathrm{zu}$ ungünstigeren Verläufen der üblicherweise weniger $\mathrm{zu}$ Metastasierung neigenden Neoplasien.

Vor dem Hintergrund der mit dem Alter steigenden Inzidenzraten der Haut- 
tumoren analog zum Inzidenzgipfel der RA in der 6. Lebensdekade sowie aufgrund der Bedeutung immunologischer Kontrollmechanismen der Tumorprogression und Metastasierung fokussiert diese Übersichtsarbeit auf den Einfluss der immunmediierten Inflammationserkrankung per se, aber auch ihrer Therapie auf das Neuerkrankungs- und Rezidivrisiko für das Melanom sowie die NMSC. Limitationen ergeben sich durch die bewusste Beschränkung auf die häufigen Tumorentitäten des BCC, SCC und des malignen Melanoms. Keine Berücksichtigung finden hier kutane Lymphome und seltenere maligne Hauttumoren (wie Merkel-Zell-Karzinom u.a.) aufgrund des Fehlens ausreichend großer valider kontrollierter Datensätze.

\section{Kriterien der berücksichtigten Literatur}

Der Übersichtsarbeit liegt eine systematische Literaturrecherche zugrunde, ohne dass der Anspruch einer wissenschaftlichen Metaanalyse der verfügbaren Daten erhoben wird.

Die Literaturübersicht berücksichtigt die unter den nachfolgenden Stichworten in der Literaturrecherche bis 30.09.2015 in den Datenbanken MEDLINE, PubMed und EMBASE publizierten Daten sowie die auf den ACR (American College of Rheumatology)- bzw. EULAR (European League Against Rheumatism)-Kongressen bis einschließlich dem EULAR-Kongress 2015 publizierten Beiträge zu dieser Thematik: Melanom, NMSC, BCC, SCC, Anti-TNF, Etanercept, Adalimumab, Infliximab, Golimumab, Certolizumab, Rituximab, Abatacept, Tocilizumab, Anakinra, Ustekinumab, Methotrexat, Leflunomid, Sulfasalazin, (Hydroxy-)Chloroquin.

Insgesamt wurden präferenziell Registerdaten aus den verschiedenen Biologikaregistern in Europa und Nordamerika aus vorpublizierten Übersichten, Originalpublikationen, Metaanalysen und Kongressbeiträgen verwendet. Darüber hinaus wurden publizierte Sicherheitsdaten der zulassungsrelevanten Biologikastudien berücksichtigt (inklusive Verlaufsdokumentation der Open-LabelExtension), während Einzelfallberichte mit begleitenden Literaturübersichten keine systematische separate Auswertung erfahren haben.

Die aufgrund des Studiendesigns bzw. der repräsentativen Patientenzahlen berücksichtigten Literaturhinweise mit den wichtigsten Studienergebnissen bezüglich der medikamentenabhängigen Risiken sowohl unter Biologika als auch konventionellen synthetischen DMARDs (csDMARD) sind in • Tab. 1 und 2 dargestellt. Nicht für alle Studien liegen dabei Angaben zur Risikoerhöhung des Auftretens von Hautneoplasien bei den therapierten RA-Patienten gegenüber der Gesamtbevölkerung als standardisierte Inzidenzratio (SIR) vor, in einigen Publikationen wird daher das Tumorrisiko als Hazard-Ratio (HR), Odds-Ratio (OR), oder InzidenzratenRatio (IRR) aus dem Vergleich der Biologika-therapierten Gruppe zu Patienten unter einer csDMARD-Therapie angegeben. Für die Biologika ist dabei zurzeit die Datenlage am besten für die TNF-Blocker, während für andere Therapieprinzipien (B-Zell-Depletion, Interleukin-1- und -6-Blockade, Kostimulationsblockade) die verfügbaren Daten vorwiegend aus den Open-LabelPhasen der randomisierten klinischen Studien stammen und Registerdaten bisher nur in geringem Umfang vorliegen (• Tab. 2). Die Daten zu konventionellen csDMARDs, insbesondere zur NichtMTX (Methotrexat)-Pharmakotherapie, sind ebenfalls spärlich (• Tab. 2).

\section{Malignes Melanom}

Die Ergebnisse der Literaturrecherche für das maligne Melanom sind in • Tab. 1 detailliert dargestellt. Repräsentativ soll nachfolgend auf die gemeinsame Auswertung der europäischen Biologikaregister zum therapieassoziierten $\mathrm{Me}$ lanomrisiko ausführlicher eingegangen werden [14]. Die Auswertungen der Register aus Portugal, Deutschland, UK, Dänemark und Schweden mit über 55.000 Patienten und einem Follow-up von 3 Mio. Patientenjahren weisen für die Biologika-naive RA-Population eine Inzidenzrate von insgesamt 160 Fällen/300.000 Patientenjahre aus. Hieraus ergab sich im Vergleich zur allgemeinen
Bevölkerung in der ausgewerteten Gesamtpopulation mit einer SIR von 1,1 bei länderspezifischen Schwankungen zwischen 0 und 1,1 kein Hinweis auf ein erhöhtes Melanomrisiko.

\section{I) Wichtig ist eine Beachtung individueller Patientenrisiken}

In der Gruppe der mit TNF-Blockern therapierten Patienten wurden Daten aus Schweden, Dänemark, UK, Deutschland, Tschechien, der Schweiz, Italien und Portugal berücksichtigt und eine Inzidenzrate von 106 Fällen/242.000 Behandlungsjahren mit einer SIR von 1,2 ermittelt. Die Inzidenzraten-Ratio (IRR) der Patientengruppe unter TNF-Blocker-Therapie in Relation zu der mit synthetischen DMARDs behandelten ergab einen Wert von IRR = 1,1 (95 \%-Konfidenzintervall: 0,8-1,6). Auch wenn damit eine sehr große europäische Studie keinen Beleg für ein erhöhtes Melanomrisiko nach TNF-Inhibitor-Exposition im Vergleich zur Gruppe Biologika-naiver Patienten ergeben hat, so erlaubt die Datenlage dennoch keinen sicheren Risikoausschluss. Dies beruht u. a. darauf, dass länderspezifische Unterschiede zu verzeichnen waren, die z. B. für Schweden und Dänemark zu SIR-Raten > 1,3 führten. Hierfür könnten unterschiedliche UV-Expositionen und -Suszeptibilitäten der $\mathrm{Pa}$ tienten, aber auch ein möglicher Bias durch unterschiedliche Tumorerfassungen (systematisches zentrales Tumorregister vs. Arztmeldebogensystem) bzw. Vernetzungsgrade der jeweiligen Register mit anderen epidemiologischen $\mathrm{Da}$ tenquellen verantwortlich sein.

Diese vorsichtige Interpretation der hier aufgrund der Aktualität und Repräsentativität großer Patientenzahlen aus Europa ausführlicher dargestellten Registerstudie, die bisher ausschließlich als Kongressbeitrag [14] publiziert ist, wird durch die Ergebnisse der bereits als Vollpublikationen vorliegenden Studien zu diesem Thema (•Tab. 1) gestützt. Die Publikationen weisen durchgängig ein erhöhtes Neuerkrankungsrisiko für Melanome unter einer TNF-BlockerTherapie aus, obgleich ein Signifikanzniveau für diesen generellen Trend nur in 
Z Rheumatol 2016 · 75:32-40 DOI 10.1007/s00393-015-0037-3

(c) Springer-Verlag Berlin Heidelberg 2016

\section{H. Burkhardt · P. Weisenseel · M. A. Radtke · K. Krüger}

\section{Hautmalignome und immunmodulierende Antirheumatikatherapie}

\section{Zusammenfassung}

Es bestehen Unsicherheiten in der Einschätzung des mit der therapeutischen Immunmodulation der rheumatoiden Arthritis (RA) verbundenen Risikos einer Beeinträchtigung der protektiven Tumorimmunität. Insbesondere für Hautneoplasien sind gesteigerte Inzidenzraten aus der Transplantationsmedizin in Assoziation zur therapeutischen Immunsuppression bekannt. Diese Daten erlauben jedoch aufgrund der zur Transplantaterhaltung erforderlichen stärkeren Immunkompromittierung des Empfängers keine unmittelbare Übertragung auf die mit der RA-Therapie verbundenen Risiken der Entstehung bzw. Rezidivierung von Melanomen oder nichtmelanotischem Hautkrebs (NMSC), speziell des spinozellulären- und Basalzellkarzinoms. Die Literaturübersicht stellt die aktuelle Datenlage über den Zusammenhang zwischen der RA und ihrer Pharmakotherapie mit konventionellen synthetischen (cs) und biologischen krankheitsmodifizierenden Antirheumatika (DMARD) auf der Basis einer systematischen Recherche dar. Die Inzidenzraten für Melanome sind bei Biologika-naiven RA-Patienten im Vergleich zur Gesamtbevölkerung nicht erhöht; dagegen ist für NMSC von einer Risikoverdopplung auszugehen. Eine Biologikatherapie mit TNFInhibitoren beeinflusst das NMSC-Risiko nicht bzw. nur geringgradig, während das Melanomneuerkrankungsrisiko gegenüber einer Behandlung mit konventionellen
csDMARD im Trend erhöht ist, ohne in allen Studien Signifikanzniveau zu erreichen. Die Datenlage zu nicht-TNF-blockierenden Biologika ist derzeit noch sehr spärlich und erlaubt bei bisher fehlenden Sicherheitssignalen keine validen Schlussfolgerungen zum Hauttumorrisiko. Eine Beachtung individueller Risiken, Empfehlungen einer suffizienten UV-Protektion und ein regelmäßiges Hautscreening dienen der Sicherheit der DMARD-Therapie.

\section{Schlüsselwörter}

Melanom $\cdot$ Basalzellkarzinom · Spinozelluläres Karzinom $\cdot$ Disease modifying antirheumatic drugs $\cdot$ Biologika

\section{Malignancies of the skin and immunomodulatory antirheumatic therapy}

\section{Abstract}

For the clinical practice there is uncertainty as to what degree the therapeutic immunomodulation of rheumatoid arthritis (RA) is associated with a weakening of protective tumor immunity. Neoplasms of the skin in particular are known to exhibit increased incidence rates in association with therapeutic immunosuppression in transplantation medicine; however, the immunosuppression required for the prevention of allogenic graft rejection is much more intensive and thus not directly transferable to the potential risks for an onset or relapse of melanoma or non-melanoma skin cancers (NMSC), e. g. spinocellular and basal cell carcinomas in association with the antirheumatic treatment of RA. This review covers the association of RA and its pharmacotherapy with conventional synthetic (cs) and biologic disease-modifying antirheumatic drugs (DMARD) on the basis of a systematic literature search. The incidence rates of NMSC are twice as high in biologicnaive RA patients compared to the general population, whereas the respective incidence rates for melanoma do not differ. A biologic treatment with tumor necrosis factor (TNF) blockers compared with administration of csDMARD only has a minor, if any effect on the increase of NMSC risk but is associated with a trend towards an elevated incidence rate of new onset melanoma although significance level was not reached in all of the reviewed studies. The data on non-TNF blocking biologics is sparse. Accordingly, it is inappropriate to draw any strong conclusions on potentially associated skin cancer risks from the present lack of safety signals. The consideration of individual risk factors, recommendations on sufficient UV protection and regular skin monitoring may serve to improve the safety of DMARD therapy in RA.

\section{Keywords}

Melanoma $\cdot$ Basal cell carcinoma $\cdot$ Spinocellular carcinoma - Disease modifying antirheumatic drugs · Biologics
3 von 11 Studien (in $\bullet$ Tab. 1 hervorgehoben) erreicht wird. In der Bewertung der Erhöhung des relativen Gesamtrisikos ist jedoch zu berücksichtigen, dass die mit der Biologikatherapie assoziierte absolute Fallzahlerhöhung sehr gering ist (in [12]: ca. 20/100.000 Behandlungsjahre), sodass erst nach der 1-jährigen Behandlung mehrerer 1000 RA-Patienten mit einer zusätzlich therapieinduzierten Melanomerkrankung gerechnet [11] werden muss und die generell positive Nutzen-Risiko-Bilanz der TNFBlockade nicht beeinträchtigt erscheint.
Wichtig ist jedoch eine erhöhte Vigilanz im Patientenmonitoring und eine Beachtung individueller Patientenrisiken. Für das Szenario der RA-Therapieinitiierung nach bereits vorbehandelter Melanomerkrankung liefert eine im britischen Biologikaregister (BSRBR) dokumentierte 18\%ige Rezidivrate des Malignoms (in 3 von 17 Fällen) unter TNF-Blockern im Vergleich zur kompletten Rezidivfreiheit csDMARD-behandelter Patienten ( $\mathrm{n}=$ 10) einen Warnhinweis [15].

Für die anderen Biologika liegen noch insgesamt spärliche Daten vor, jedoch sind in der gemeinsamen Auswertung der europäischen Biologikaregister (• Tab. 1; [14]) 5000 Patienten unter RituximabTherapie aus Schweden, Dänemark, UK, Deutschland, Frankreich und der Schweiz berücksichtigt. Es wurden insgesamt 14 Melanomneuerkrankungen in 29.000 Patientenjahren registriert und eine SIR von 1,3, die jedoch unter Berücksichtigung des $95 \%$-Konfidenzintervalls statistisch nicht signifikant war, kalkuliert. Präliminare Daten wurden auch zu Tocilizumab auf einer Basis von $5800 \mathrm{~Pa}$ tientenjahren mit 5 Fällen und für Aba- 
Tab. 1 Therapie der rheumatoiden Arthritis und assoziiertes Melanomrisiko

\begin{tabular}{|c|c|c|c|c|c|c|}
\hline $\begin{array}{l}\text { Referenz [Num- } \\
\text { mer im Litera- } \\
\text { turverzeichnis] }\end{array}$ & Datenquelle & DMARD & $\begin{array}{l}\text { Patientenzahl } \\
\text { (Patientenjahre) }\end{array}$ & $\begin{array}{l}\text { Inzidenzrate/ } \\
100 \text { Patienten- } \\
\text { jahre (95\%-KI) }\end{array}$ & Risiko (95 \%-KI) & $\begin{array}{l}\text { Standardisierte } \\
\text { Inzidenzrate } \\
(95 \%-K \mathrm{I})\end{array}$ \\
\hline \multirow[t]{3}{*}{ Askling et al. [4] } & $\begin{array}{l}\text { Swedish: Early } \\
\text { Arthritis Register }\end{array}$ & NR & 3703 (13.292) & NR & NR & $0,9(0,2-2,2)$ \\
\hline & Inpatient Register & NR & $53.067(297.102)$ & & & $1,19(0,99-1,42)$ \\
\hline & $\begin{array}{l}\text { Anti-TNF Postmar- } \\
\text { keting Cohort }\end{array}$ & ETA, ADA, INF & $4160(9715)$ & & & $0,3(0,0-1,8)$ \\
\hline \multirow{2}{*}{$\begin{array}{l}\text { Greenberg et al. } \\
\text { [5] }\end{array}$} & \multirow[t]{2}{*}{ CORRONA } & Anti-TNF naiv & $4153(6805)$ & 0 (NR) & \multirow[t]{2}{*}{ NR } & \multirow[t]{2}{*}{ NR } \\
\hline & & Anti-TNF & $4651(8690)$ & 0,035 (NR) & & \\
\hline \multirow[t]{6}{*}{$\begin{array}{l}\text { Wolfe und } \\
\text { Michaud [6] }\end{array}$} & \multirow{6}{*}{$\begin{array}{l}\text { National Data } \\
\text { Bank for } \\
\text { Rheumatic } \\
\text { Diseases USA }\end{array}$} & NR & 13.869 (48.795) & $\begin{array}{l}0,0656 \\
(0,0449-0,0926)\end{array}$ & \multirow{2}{*}{$\begin{array}{l}\text { Biologika vs. } \\
\text { Nicht-Biologika: OR } 2,3 \\
(0,9-5,4) \\
p=0,070\end{array}$} & $1,7(1,3-2,3)$ \\
\hline & & \multirow[t]{5}{*}{ Davon: Biologika } & 6597 & NR & & \multirow[t]{5}{*}{ NR } \\
\hline & & & INF & & OR $2,6(1,0-6,7)$ & \\
\hline & & & ETA & & OR $2,4(1,0-5,8)$ & \\
\hline & & & $\mathrm{ADA}$ & & OR $0,8(0,1-6,6)$ & \\
\hline & & & ANA & & OR 4,2 $(0,9-20,0)$ & \\
\hline Askling [7] & ARTIS & Anti-TNF & 6604 (NR) & NR & $\begin{array}{l}\text { Biologika vs. Nicht-Bio- } \\
\text { logika: } \\
R R 1,7(1,0-2,9)\end{array}$ & NR \\
\hline Mariette et al. [8] & $\begin{array}{l}\text { Metaanalyse } \\
\text { von Wolfe und } \\
\text { Michaud (2007) } \\
\text { und Askling (2009) }\end{array}$ & S.o. & S. o. & S.o. & $\begin{array}{l}\text { Anti-TNF vs. Nicht-Bio- } \\
\text { logika: } \\
\text { 1,79 }(0,92-2,67)\end{array}$ & S.o. \\
\hline Mercer et al. [9] & BSRBR & csDMARD & 3771 (13.315) & $0,07(0,03-0,13)$ & NR & $2,05(0,94-3,90)$ \\
\hline $\begin{array}{l}\text { Burmester et al. } \\
\text { [10] }\end{array}$ & $\begin{array}{l}\text { Metaanalyse von } \\
36 \text { RA-Studien }\end{array}$ & Anti-TNF (ADA) & 14.109 (23.942) & $<0,1$ & NR & $1,5(0,84-2,47)$ \\
\hline \multirow[t]{2}{*}{$\begin{array}{l}\text { Raaschou et al. } \\
{[11]}\end{array}$} & \multirow{2}{*}{$\begin{array}{l}\text { ARTIS und } \\
\text { vernetzte } \\
\text { nationale } \\
\text { schwedische } \\
\text { klinische } \\
\text { Gesundheits- und } \\
\text { demografische } \\
\text { Register }\end{array}$} & Biologika-naiv & $42.198(203.345)$ & 0,056 & $\begin{array}{l}\text { Invasiv } \\
1,2(0,9-1,5) \\
\text { In situ } \\
1,2(0,9-1,7)\end{array}$ & \multirow[t]{2}{*}{ NR } \\
\hline & & Anti-TNF & $11.343(57.223)$ & 0,068 & $\begin{array}{l}\text { Invasiv } \\
1,5(1,1-2,5) \\
\text { In situ } \\
1,1(0,5-2,1)\end{array}$ & \\
\hline \multirow[t]{2}{*}{ Dreyer et al. [12] } & \multirow[t]{2}{*}{ DANBIO Register } & Anti-TNF-naiv & 3812 (NR) & & \multirow{2}{*}{$\begin{array}{l}\text { Biologika vs. } \\
\text { Anti-TNF-naiv: } \\
\text { HR 1,54 }(0,37-6,34)\end{array}$} & $1,00(0,32-3,11)$ \\
\hline & & Anti-TNF (gesamt) & 3347 (NR) & & & $1,57(0,70-3,49)$ \\
\hline Gross et al. [13] & CORRONA & NR & $19.260(53.864)$ & $0,03(0,02-0,05)$ & NR & NR \\
\hline \multirow[t]{5}{*}{ Mercer et al. [14] } & \multirow{5}{*}{$\begin{array}{l}\text { Gemeinsame } \\
\text { Auswertung } \\
\text { gepoolter } \\
\text { Datensätze } \\
\text { verschiedener } \\
\text { europäischer } \\
\text { Biologikaregister }\end{array}$} & Biologika-naiv & $55.119(3.000 .011)$ & \multirow[t]{5}{*}{ NR } & \multirow{5}{*}{$\begin{array}{l}\text { Anti-TNF vs. } \\
\text { Nicht-Biologika: } \\
\text { IRR } 1,1(0,8-1,6)\end{array}$} & 1,1 (NR) \\
\hline & & Anti-TNF & $41.753(241.814)$ & & & 1,2 (NR) \\
\hline & & MAB & $5221(29.619)$ & & & 1,3 (NR) \\
\hline & & $\mathrm{ABA}$ & $1220(4858)$ & & & $1,3(0,2-7,6)$ \\
\hline & & TOC & 2151 (5798) & & & $2,7(0,6-10,1)$ \\
\hline
\end{tabular}

Statistisch signifikante Ergebnisse sind durch kursiv hervorgehobenen Text markiert.

KI 95 \%-Konfidenzintervall, NR nicht berichtet („non reported"), INF Infliximab, ETA Etanercept, ADA Adalimumab, ANA Anakinra, MAB Mabthera, Rituximab, ABA Abatacept, TOC Tocilizumab, OR Odds-Ratio, HR Hazard-Ratio, IRR Inzidenzraten-Ratio, CORRONA Consortium of Rheumatology Researchers of North America, ARTIS Anti-Rheumatic Therapies in Sweden, BSRBR British Society for Rheumatology Biologics Register, DMARD "disease-modifying anti-rheumatic drugs", $R A$ rheumatoide Arthritis 


\section{Leitthema}

Tab. 2 Therapie der rheumatoiden Arthritis und assoziiertes Risiko für Nicht-Melanom-Hauttumoren (NMSC): spinozelluläres Karzinom (SCC) und Basalzellkarzinom (BCC)

\begin{tabular}{|c|c|c|c|c|c|c|}
\hline $\begin{array}{l}\text { Referenz } \\
\text { [Nummer im } \\
\text { Literaturver- } \\
\text { zeichnis] }\end{array}$ & Datenquelle & DMARD & $\begin{array}{l}\text { Patientenzahl } \\
\text { (Patientenjahre) }\end{array}$ & $\begin{array}{l}\text { Inzidenzrate/ } \\
100 \text { Patientenjah- } \\
\text { re (95\%-KI) }\end{array}$ & Risiko (95 \%-KI) & $\begin{array}{l}\text { Standardisierte } \\
\text { Inzidenzrate } \\
(95 \%-K I)\end{array}$ \\
\hline \multirow[t]{3}{*}{$\begin{array}{l}\text { Askling et al. [4] } \\
\text { NMSC }\end{array}$} & $\begin{array}{l}\text { Swedish: } \\
\text { Early Arthritis } \\
\text { Register }\end{array}$ & NR & 3703 (13.292) & \multirow[t]{3}{*}{ NR } & \multirow[t]{3}{*}{ NR } & $0,7(0,2-1,6)$ \\
\hline & Inpatient Register & NR & $53.067(297.102)$ & & & $1,66(1,50-1,84)$ \\
\hline & $\begin{array}{l}\text { Anti-TNF } \\
\text { Postmarketing } \\
\text { Cohort }\end{array}$ & ETA,ADA, INF & $4160(9715)$ & & & $3,6(1,8-6,5)$ \\
\hline \multirow{6}{*}{$\begin{array}{l}\text { Chakravarty } \\
\text { et al. [16] } \\
\text { NMSC }\end{array}$} & \multirow{6}{*}{$\begin{array}{l}\text { National Data Bank } \\
\text { for Rheumatic } \\
\text { Diseases USA }\end{array}$} & & \multirow[t]{6}{*}{$15.784(40.125)$} & \multirow[t]{6}{*}{ NR } & $\begin{array}{l}\text { COX-Regression } \\
\text { HR: }\end{array}$ & \multirow[t]{6}{*}{ NR } \\
\hline & & Prednison & & & $1,28(1,05-1,55)$ & \\
\hline & & MTX & & & $1,15(0,81-1,64)$ & \\
\hline & & $\begin{array}{l}\text { Leflunomid } \\
\text { (LEF) }\end{array}$ & & & $0,89(0,68-1,18)$ & \\
\hline & & TNF-Mono & & & $1,24(0,97-1,58)$ & \\
\hline & & $T N F+M T X$ & & & $1,97(1,51-2,58)$ & \\
\hline \multirow{2}{*}{$\begin{array}{l}\text { Greenberg et al. } \\
\text { [5] } \\
\text { NMSC }\end{array}$} & \multirow[t]{2}{*}{ CORRONA } & CsDMARD & $4153(6805)$ & 0,16 (NR) & \multirow{2}{*}{$\begin{array}{l}\text { Anti-TNF vs. } \\
\text { Nicht-Biologika } \\
\text { IRR } \\
1,83(0,85-3,93)\end{array}$} & \multirow[t]{2}{*}{ NR } \\
\hline & & Anti-TNF & $4651(8690)$ & 0,23 (NR) & & \\
\hline \multirow{2}{*}{$\begin{array}{l}\text { Wolfe und } \\
\text { Michaud [6] } \\
\text { NMSC }\end{array}$} & \multirow{2}{*}{$\begin{array}{l}\text { National Data Bank } \\
\text { for Rheumatic } \\
\text { Diseases USA }\end{array}$} & NR & $13.869(48.795)$ & \multirow[t]{2}{*}{ NR } & \multirow{2}{*}{$\begin{array}{l}\text { Biologika vs. } \\
\text { Nicht-Biologika: OR 1,5 } \\
(1,2-1,8)\end{array}$} & \multirow[t]{2}{*}{ NR } \\
\hline & & $\begin{array}{l}\text { Davon: } \\
\text { Biologika }\end{array}$ & $\begin{array}{l}6597 \\
\text { INF, ETA, ADA, ANA }\end{array}$ & & & \\
\hline \multirow{8}{*}{$\begin{array}{l}\text { Leombruno } \\
\text { et al. [17] } \\
\text { NMSC }\end{array}$} & \multirow{8}{*}{$\begin{array}{l}\text { Metaanalyse von } \\
15 \text { RCTs (Anti-TNF } \\
\text { in empfohlener } \\
\text { Dosierung) } \\
7 \text { RCTs (Anti-TNF in } \\
\text { hoher Dosierung) }\end{array}$} & $\begin{array}{l}\text { Anti-TNF (ge- } \\
\text { samt) }\end{array}$ & 5416 (4738) & \multirow[t]{8}{*}{ NR } & $\begin{array}{l}\text { Anti-TNF vs. Placebo } \\
1,01(0,42-2,44)\end{array}$ & \multirow[t]{8}{*}{ NR } \\
\hline & & ETA & $1981(1465)$ & & $2,02(0,43-9,31)$ & \\
\hline & & INF & $982(867)$ & & $0,61(0,11-3,42)$ & \\
\hline & & ADA & $2453(2406)$ & & $0,66(0,15-2,92)$ & \\
\hline & & $\begin{array}{l}\text { Anti-TNF (ge- } \\
\text { samt) }\end{array}$ & $1719(1325)$ & & $0,75(0,21-2,60)$ & \\
\hline & & ETA & NR & & NR & \\
\hline & & INF & $1159(1141)$ & & $0,91(0,19-4,40)$ & \\
\hline & & ADA & $560(184)$ & & $0,56(0,07-4,28)$ & \\
\hline \multirow[t]{5}{*}{$\begin{array}{l}\text { Mercer et al. [18] } \\
\text { NMSC }\end{array}$} & \multirow[t]{5}{*}{ BSRBR } & csDMARD & $3515(9058)$ & $0,24(0,15-0,36)$ & $\begin{array}{l}\text { Biologika vs. Nicht-Bio- } \\
\text { logika: }\end{array}$ & \multirow[t]{5}{*}{ NR } \\
\hline & & $\begin{array}{l}\text { Anti-TNF (ge- } \\
\text { samt) }\end{array}$ & $11.757(41.716)$ & $0,35(0,29-0,41)$ & HR $1,0(0,6-1,6)$ & \\
\hline & & ETA & NR (18.133) & $0,30(0,22-0,39)$ & HR $1,0(0,6-1,6)$ & \\
\hline & & INF & NR (11.212) & $0,55(0,42-0,71)$ & HR $1,0(0,6-1,6)$ & \\
\hline & & ADA & NR (12.371) & $0,24(0,16-0,34)$ & HR 1,0 $(0,6-1,6)$ & \\
\hline $\begin{array}{l}\text { Askling [7] } \\
\text { SCC }\end{array}$ & ARTIS & Anti-TNF & 6604 (NR) & NR & $\begin{array}{l}\text { Biologika vs. Nicht-Bio- } \\
\text { logika: } \\
\text { RR } 1,2(0,8-2,0)\end{array}$ & NR \\
\hline $\begin{array}{l}\text { Mariette et al. } \\
\text { [8] } \\
\text { NMSC }\end{array}$ & $\begin{array}{l}\text { Metaanalyse } \\
\text { von Greenberg } \\
\text { (2007), Wolfe und } \\
\text { Michaud (2007), } \\
\text { Mercer (2009) und } \\
\text { Askling (2009) }\end{array}$ & S. o. & S.o. & S. o. & $\begin{array}{l}\text { Anti-TNF vs. Nicht-Bio- } \\
\text { logika: } \\
\text { 1,45 }(1,15-1,76)\end{array}$ & S. o. \\
\hline
\end{tabular}


Tab. 2 Therapie der rheumatoiden Arthritis und assoziiertes Risiko für Nicht-Melanom-Hauttumoren (NMSC): spinozelluläres Karzinom (SCC) und Basalzellkarzinom (BCC) (Fortsetzung)

\begin{tabular}{|c|c|c|c|c|c|c|}
\hline $\begin{array}{l}\text { Referenz } \\
\text { [Nummer im } \\
\text { Literaturver- } \\
\text { zeichnis] }\end{array}$ & Datenquelle & DMARD & $\begin{array}{l}\text { Patientenzahl } \\
\text { (Patientenjahre) }\end{array}$ & $\begin{array}{l}\text { Inzidenzrate/ } \\
100 \text { Patientenjah- } \\
\text { re }(95 \%-K I)\end{array}$ & Risiko (95 \%-KI) & $\begin{array}{l}\text { Standardisierte } \\
\text { Inzidenzrate } \\
(95 \%-K I)\end{array}$ \\
\hline Mercer et al. [19] & \multirow[t]{7}{*}{ BSRBR } & csDMARD & $3523(9342)$ & $0,41(0,29-0,56)$ & $\begin{array}{l}\text { Biologika vs. Nicht-Bio- } \\
\text { logika: }\end{array}$ & \multirow{7}{*}{$\begin{array}{l}\text { NMSC (Gesamt): } \\
\text { csDMARD: } \\
\text { 1,83 (1,30-2,50) } \\
\text { Anti-TNF: } \\
\text { 1,72 }(1,43-2,04)\end{array}$} \\
\hline \multirow[t]{4}{*}{$\mathrm{BCC}$} & & $\begin{array}{l}\text { Anti-TNF (ge- } \\
\text { samt) }\end{array}$ & $11.704(43.798)$ & $0,34(0,29-0,40)$ & HR 0,95 $(0,5-1,7)$ & \\
\hline & & ETA & $5086(19.108)$ & $0,30(0,23-0,39)$ & HR 0,8 $(0,4-1,5)$ & \\
\hline & & INF & $3663(11.700)$ & $0,55(0,38-0,65)$ & HR $1,5(0,8-2,9)$ & \\
\hline & & ADA & 5035 (12.991) & $0,26(0,18-0,37)$ & HR 0,7 $(0,4-1,5)$ & \\
\hline \multirow[t]{2}{*}{ SCC } & & csDMARD & $3523(9342)$ & $0,043(0,01-0,11)$ & \multirow{2}{*}{$\begin{array}{l}\text { Biologika vs. } \\
\text { Nicht-Biologika: } \\
\text { HR 1,16 }(0,35-3,84)\end{array}$} & \\
\hline & & $\begin{array}{l}\text { Anti-TNF (ge- } \\
\text { samt) }\end{array}$ & 11.704 (43.798) & $0,053(0,033-0,079)$ & & \\
\hline $\begin{array}{l}\text { Burmester et al. } \\
\text { [10] NMSC }\end{array}$ & $\begin{array}{l}\text { Metaanalyse von } \\
36 \text { RA-Studien }\end{array}$ & Anti-TNF (ADA) & $14.109(23.942)$ & 0,2 (NR) & NR & $1,39(1,19-1,60)$ \\
\hline \multirow{2}{*}{$\begin{array}{l}\text { Dreyer et al. [12] } \\
\text { NMSC }\end{array}$} & \multirow{2}{*}{ DANBIO-Register } & Anti-TNF-naiv & 3812 (NR) & \multirow[t]{2}{*}{ NR } & \multirow{2}{*}{$\begin{array}{l}\text { Biologika vs. } \\
\text { Nicht-Biologika: } \\
\text { HR 1,10 }(0,69-1,76)\end{array}$} & $1,92(1,42-2,59)$ \\
\hline & & Anti-TNF(gesamt) & 3347 (NR) & & & $1,76(1,26-2,46)$ \\
\hline $\begin{array}{l}\text { Gross et al. [13] } \\
\text { NMSC }\end{array}$ & CORRONA & NR & $19.260(53.864)$ & $0,20(0,17-0,24)$ & NR & NR \\
\hline $\begin{array}{l}\text { Raaschou et al. } \\
\text { [20] }\end{array}$ & $\begin{array}{l}\text { ARTIS und } \\
\text { vernetzte }\end{array}$ & & & & & NR \\
\hline \multirow[t]{2}{*}{$\mathrm{BCC}$} & nationale & Biologika-naiv & 41.735 (200.615) & 0,806 (NR) & \multirow{2}{*}{$\begin{array}{l}\text { Biologika vs. } \\
\text { Nicht-Biologika } \\
\text { HR: } 1,3(1,3-1,5)\end{array}$} & \\
\hline & $\begin{array}{l}\text { klinische } \\
\text { Gesundheits- und }\end{array}$ & Anti-TNF & 8039 (34.601) & 0,630 (NR) & & \\
\hline \multirow[t]{2}{*}{ SCC } & demografische & Biologika-naiv & $44.032(235.125)$ & 0,369 & \multirow{2}{*}{$\begin{array}{l}\text { Biologika vs. } \\
\text { Nicht-Biologika } \\
\text { HR: } 1,3(1,1-1,6)\end{array}$} & \\
\hline & Register & Anti-TNF & $11.484(70.335)$ & 0,273 (NR) & & \\
\hline \multirow{4}{*}{$\begin{array}{l}\text { Gottenberg } \\
\text { et al. [23] } \\
\text { NMSC }\end{array}$} & $\begin{array}{l}\text { Französische } \\
\text { Register }\end{array}$ & & & & \multirow[t]{4}{*}{ NR } & \multirow[t]{4}{*}{ NR } \\
\hline & AIR & $M A B$ & $1980(6844)$ & 0,2 (NR) & & \\
\hline & ORA & $A B A$ & $1024(2882)$ & 0,5 (NR) & & \\
\hline & REGATE & TOC & $1503(1552)$ & 0,19 (NR) & & \\
\hline
\end{tabular}

Statistisch signifikante Ergebnisse sind durch kursiv hervorgehobenen Text markiert.

KI 95 \%-Konfidenzintervall, NR nicht berichtet(„,non reported“), RCT randomisierte kontrollierte klinische Studie („,randomized controlled clinical trial“), INF Infliximab, ETA Etanercept, ADA Adalimumab, ANA Anakinra, ABA Abatacept, OR Odds-Ratio, HR Hazard-Ratio, IRR Inzidenzraten-Ratio, DANBIO Danish Registry for Biologic Therapies in Rheumatology, CORRONA Consortium of Rheumatology Researchers of North America, ARTIS Anti-Rheumatic Therapies in Sweden, BSRBR British Society for Rheumatology Biologics Register, AIR Autoimmunité et Rituximab, ORA Orencia et Rheumatoid Arthritis, REGATE Registry-RoActemra, $D M A R D$ "disease-modifying anti-rheumatic drugs", RA rheumatoide Arthritis

tacept mit 2 Fällen/4800 Patientenjahre berichtet [14]. Aus diesen Inzidenzraten ergab sich rechnerisch durch Vergleich mit der Gesamtbevölkerung eine SIR von 2,7 (95 \%-Konfidenzintervall: 0,6-10,1) für Tocilizumab und 1,3 (95 \%-Konfidenzintervall: 0,2-7,6) für Abatacept, die aber jeweils unter Berücksichtigung der 95 \%-Konfidenzintervalle kein Signifikanzniveau erreichten [14]. Insgesamt liegt zu diesem Biologika derzeit keine ausreichende Datenlage vor, um das
Risiko bezüglich der Melanominzidenz valide abschätzen zu können.

\section{Nicht-Melanom-Neoplasien der Haut: Basalzellkarzinom und spinozelluläres Karzinom}

Für die NMSC liegen die in • Tab. 2 gelisteten Studien vor, die in weitgehender Übereinstimmung ein erhöhtes Risiko für das Auftreten in der RA-Population im Vergleich zur Gesamtbevölkerung ausweisen. Lediglich in 2 Studien $[19,20]$ sind die NMSC-Entitäten des SCC bzw. BCC separat analysiert. Hierbei finden sich keine Hinweise auf tumorspezifische Unterschiede für das in der RA-Population generell erhöhte NMSC-Risiko. Insgesamt ergibt sich aus den in der $\bullet$ Tab. 2 berücksichtigten Studien zum NMSCNeuerkrankungsrisiko für RA-Patienten unter biologischer DMARD-Therapie ein uneinheitliches Bild mit Studien, die eine erhöhte Inzidenzrate unter Anti-TNFTherapie belegen $[4,6,8,16,20]$, Studien mit einem Trend für ein erhöhtes 
Tab. 3 Exemplarische Empfehlungen des American College of Rheumatology

Jahr des Erscheinens

Empfehlung

$2012[28]$

NMSC (therapiert) > 5 Jahre: Therapie mit jedem Biologikum möglich

NMSC (therapiert) $<5$ Jahre: Therapie mit Rituximab

Melanom (therapiert): Therapie mit Rituximab

2015 [29] NMSC unbehandelt oder therapiert: präferenzieller Einsatz einer konventionellen synthetischen DMARD-Kombination oder eines Nicht-TNF-Biologikums gegenüber TNF-Inhibitor

NMSC Nicht-Melanom-Hauttumoren, DMARD "disease-modifying anti-rheumatic drugs"

Risiko unter TNF-Blockern ohne statistische Signifikanz $[5,7,12]$ und Analysen, die keine Evidenzen einer NMSC-Assoziation zur TNF-Blocker-Therapie zeigen $[17,19]$.

Detaillierter soll auf die Studie von Raschou et al. [20] eingegangen werden, die an $>8000$ mit Anti-TNF- und $>40.000$ mit csDMARD behandelten RA-Patienten unter Hinzuziehung von Bevölkerungsdaten ( $\mathrm{N}=300.000$ Individuen) aus Gesundheitsregistern ausgewertet wurden. Der Vorteil der auf dem ACR-Kongress publizierten Studie war die Verknüpfung der im schwedischen Biologikaregister (Artis) verfügbaren Verlaufsdokumentationen mit den in anderen nationalen Zentralregistern erfassten Daten (Populationsregister, Patientenregister, Krebsregister, Arzneimittelverordnungsregister und Todesfallregister). Es wurden dabei die Daten aus den Zeiträumen zwischen 1999 und 2012 berücksichtigt und die jeweiligen HR durch COXRegressionsanalyse unter Berücksichtigung vielfältiger demografischer Daten, hautbezogener Variablen (Vorgeschichte bezüglich NMSC, Melanom, benigne dermatologische Komorbiditäten, nichtRA-bezogene Immunsuppression) und anderer Komorbiditäten (andere Malignome, alloplastischer Gelenkersatz, chronisch obstruktive Lungenerkrankung (chronic obstructive lung desease, COLD), koronare Herzkrankheit, Diabetes) als Kovariaten ausgewertet. Für die Allgemeinbevölkerung standen Daten aus $>2$ Mio. Personenjahren, für die Biologika-naiven Patienten aus > 200.000 Patientenjahren und für die Individuen unter TNF-Inhibitoren aus $>34.000$ Patientenjahren zur Verfügung. Eine Auswertung erfolgte für das SCC gesamt sowie differenziert nach invasiven und nichtinvasiven Verläufen und für das BCC. Insgesamt ergab sich dabei für das SCC eine gegenüber der Gesamtpopulation knapp verdoppelte HR ohne wesentliche Unterschiede zwischen invasivem und nichtinvasivem SCC während für das BCC die HR mit 1,3 (95 \%-Konfidenzintervall: 1,3-1,5) nur leicht erhöht war. Im Vergleich zur Biologika-naiven Population wurde für die mit TNF-Inhibitoren behandelten Patienten ein leicht erhöhtes SCC-Gesamtrisiko ( $\mathrm{HR}=1,3$, 95 \%-Konfidenzintervall: 1,1-1,6) ermittelt. Diese Risikoerhöhung unter AntiTNF-Therapie betraf ausschließlich das nichtinvasive SCC. Unter Berücksichtigung der Zeitdauer des Auftretens der Karzinome unter TNF-Blockade ergab sich dabei innerhalb der ersten 6 Monate nach Initiierung eine erhöhte $\mathrm{HR}=2,1$ (95 \%-Konfidenzintervall: 1,1-4,0) mit abnehmender Tendenz bei zunehmender Therapiedauer (5 Jahre: $\mathrm{HR}=1,2$; 95 \%-Konfidenzintervall: 1,0-1,6). Hier ist denkbar, dass neben einer tatsächlich initial gesteigerten Rate auch eine vermehrte Detektionsrate bereits vor AntiTNF-Therapie prävalenter Karzinome durch ein intensiviertes Patientenmonitoring nach Biologikainitiierung eine Rolle spielt. Für das BCC ergab sich im Vergleich der RA-Patienten unter TNFBlocker-Therapie mit der Kontrollgruppe unter konventioneller synthetischer DMARD-Behandlung eine leichte Erhöhung des Risikos (HR = 1,2; 95 \%Konfidenzintervall: 1,0-1,4).

Zusammenfassend sprechen die Daten für ein verdoppeltes Risiko des Auftretens eines SCC in einer Biologikanaiven RA-Population gegenüber der Gesamtbevölkerung mit einem kurzfristigen zusätzlichen Anstieg der HR nach TNF-Inhibitor-Initiierung, evtl. begünstigt durch die gesteigerte Neuentdeckungsrate im Rahmen der mit dem Therapiewechsel intensivierten Patientenüberwachung. Für das BCC ergibt sich ein gegenüber der Gesamtbevölkerung marginal erhöhtes Risiko in der Biologika-naiven RA-Population ohne nennenswerten Anstieg unter TNF-Inhibitor-Therapie. Insgesamt stellt daher die RA per se - ggf. auch im Zusammenhang mit der konventionellen synthetischen DMARD-Therapie - einen stärkeren Risikofaktor für das Auftreten von NMSC dar als die TNF-Inhibitor-Therapie. So spricht die Studie von Chakravarty et al. [16] für eine NMSC-Risikoerhöhung durch MTX in der Kombination mit TNF-Inhibitoren und identifiziert in der multivariaten Analyse Prednison als unabhängigen Risikofaktor, während MTX bzw. Leflunomid in Monotherapie ohne Einfluss waren.

\section{》) RA ist ein stärkerer Risikofaktor für NMSC als TNF- Inhibitor-Therapie}

In diesem Zusammenhang ist ein Kongressbeitrag über die Auswertung von Medicare-Daten aus den Jahren 2006 bis 2011 mit der Identifizierung von 5994 RA-Patienten mit einem vordiagnostizierten NMSC, die im Beobachtungsintervall 847 NMSC-Zweiterkrankungen entwickelten [21], interessant. In dieser Auswertung konnte gezeigt werden, dass eine fortgesetzte MTXTherapie von länger als 1 Jahr Dauer zu einer signifikanten Erhöhung der HR für das Auftreten eines Zweittumors führte (1,24, $95 \%$-KI: 1,03-1,49). Ein hypothetisches Erklärungsmodell könnte ein photosensibilisierender Effekt von MTX darstellen, da gezeigt werden konnte, dass die Anwendung photosensibilisierender Agenzien mit einem erhöhten Risiko für das Auftreten von NMSC assoziiert ist [22]. Die oben erwähnte MedicareStudie [21] ergab auch, dass eine TNFInhibitor-Therapie mit einem numerisch leicht erhöhten, aber statistisch nicht signifikanten NMSC-Risiko behaftet war. Es waren auch keine signifikanten As- 
soziationen zu einer Rituximab- oder Abatacept-Therapie detektierbar [23].

\section{Hautkrebsrisiko unter Immunmodulation bei Psoriasispatienten}

Biologika sind in der Dermatologie neben den Hauptindikationen der Psoriasis und Psoriasisarthritis (PsA) auch für die Hidradenitis suppurativa (Adalimumab) und die Urtikaria (Omalizumab: ein monoklonaler Anti-IgE-Antikörper) zugelassen. Für 3 TNF-Blocker (Adalimumab, Etanercept, Infliximab) sowie Antikörper gegen Interleukin-12/23 (Ustekinumab) und seit Kurzem auch Interleukin-17A (Secukinumab) besteht eine Zulassung für die Therapie sowohl der Psoriasis als auch der PsA. Die Zulassung der TNFBlocker Golimumab und Certolizumab ist auf das Indikationsgebiet der PsA beschränkt. Psoriasispatienten unterscheiden sich von RA-Patienten in gewissen Aspekten, die Einfluss auf das Hautkrebsrisiko haben: Da sich die Psoriasis durch UV-Exposition in den allermeisten Fällen bessert, setzen sich viele Patienten in der Freizeit und im Urlaub über viele Jahre/Jahrzehnte regelmäßig der natürlichen Sonne oder Höhensonnengeräten aus oder werden mit UV-Licht behandelt. Dies ist eine mögliche Erklärung dafür, dass die NMSC-Rate bei Psoriasispatienten im Vergleich zur Allgemeinbevölkerung leicht erhöht ist.

\section{》) Die NMSC-Rate ist bei Psoriasispatienten leicht erhöht}

Eine längerfristige Therapie mit Immunsuppressiva und/oder Biologika kann das Risiko für Melanome und/oder NMSC nochmals erhöhen. Hierbei gibt es jedoch deutliche Unterschiede zwischen den einzelnen Therapien [24, 25].

In einer holländischen prospektiven Beobachtungsstudie von Patienten unter TNF-Blockern entwickelten Psoriasispatienten früher und zahlreicher NMSC im Vergleich zu RA-Patienten [26]. Zu Adalimumab, Etanercept und Ustekinumab liegen publizierte, kontrollierte Langzeitstudien über bis zu 5 Jahre bei Psoriasis vulgaris vor. Hierbei wurde weder kurz- fristig noch langfristig ein erhöhtes Risiko für die Entwicklung von Hautkrebs beobachtet. Auch im deutschen Psoriasisregister PsoBest konnten keine signifikanten Signale für ein erhöhtes Risiko identifiziert werden [27].

\section{Schlussfolgerungen}

In der zusammenfassenden Bewertung der aktuellen wissenschaftlichen Datenlage kann die Schlussfolgerung gezogen werden, dass bei RA-Patienten das Risiko, an einem Hauttumor zu erkranken, erhöht ist. Insgesamt sind die unter TNF- $\boldsymbol{\alpha}$-Hemmern beobachteten erhöhten Inzidenzen von Hauttumoren im Vergleich zu synthetischen konventionellen DMARDs nicht als alarmierende Sicherheitssignale zu deuten. Ein zurückhaltender Einsatz wird allerdings für den Fall einer positiven Hauttumoranamnese - insbesondere bei vorbekanntem Melanom - seitens der Fachgesellschaften empfohlen (s. ACR in • Tab. 3). Die Präferenzempfehlungen für alternative, nicht-TNF-blockierende DMARDs sind dabei weniger durch die Studienlage, die derzeit für diese Substanzen in der RA noch relativ dünn ist, gestützt, als vielmehr durch theoretische Überlegungen zu den potenziell aufgrund ihrer Wirkmechanismen zu erwartenden Beeinträchtigungen der protektiven Tumorimmunität begründet.

\section{Fazit für die Praxis}

- Für den praktischen Umgang ist vor Beginn und im weiteren Verlauf einer Behandlung mit synthetischen oder biologischen DMARDs eine besondere Aufmerksamkeit von Patient und Arzt für das Vorliegen von Hautveränderungen erforderlich.

- Eine regelmäßige, z. B. jährliche dermatologische Vorsorgeuntersuchung unter einer Biologikatherapie erscheint sinnvoll. Eine entsprechende Empfehlung inklusive eines Konsensus zu den Screeningintervallen wird derzeit von der Fachgesellschaft erarbeitet.

- Für die ersten 6 Monate einer Biologikatherapie ist eine besondere Aufmerksamkeit geboten.
- Generell sollten alle Patienten mit entzündlich rheumatischen Gelenkerkrankung auf die Anwendung eines suffizienten Sonnenschutzes hingewiesen werden.

- Unklare Hautveränderungen sollten immer eine fachdermatologische Abklärung erfahren.

\section{Korrespondenzadresse

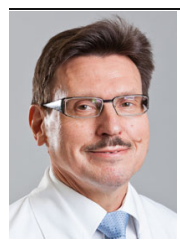 \\ Prof. Dr. H. Burkhardt \\ Abteilung Rheumatologie \& Fraunhofer IME, Projektgruppe Translationale Medizin und Pharmakologie Universitätsklinikum Frankfurt, Medizinische Klinik II, Goethe Universität Frankfurt am Main Theodor-Stern-Kai 7, 60590 Frankfurt am Main, Deutschland harald.burkhardt@kgu.de}

\section{Einhaltung ethischer Richtlinien}

Interessenkonflikt. Dieses Manuskript entstand auf Basis einer von Roche und Chugai unterstützten Arbeitsgruppe (Systemerkrankung RA), aber ohne jegliche Einflussnahme der Firmen auf das Ergebnis oder die Publikation. H. Burkhardt: Referentenhonorare und Beraterverträge: Abbvie, BMS, Chugai, Janssen, Novartis, MSD, Pfizer, Roche, UCB. P. Weisenseel: Referentenhonorare und Beraterverträge: Abbvie, Biogen Idec, Celgene, Dexcel, Eli Lilly, Janssen, Leo, Medac, Novartis, MSD. M.A. Radtke: Referentenhonorare und Beraterverträge sowie die Durchführung sowie klinischer oder versorgungswissenschaftlicher Studien: Abbvie, Amgen, Biogen, BMS, Boehringer-Ingelheim, Celgene, Chugai, Galderma, Janssen-Cilag, La RochePosay, Leo-Pharma, Lilly, Medac, Mundipharma, MSD, Novartis, Pfizer, Sandoz, UCB. K. Krüger: Referentenhonorare und Beraterverträge: Abbvie, BMS, Chugai, Janssen, Medac. MSD, Mundipharma, Pfizer, Roche, UCB.

Dieser Beitrag beinhaltet keine Studien an Menschen oder Tieren.

\section{Literatur}

1. Pflugfelder A, Kochs C, Blum A et al (2013) Malignant melanoma S3-guideline "diagnosis, therapy and follow-up of melanoma". J Dtsch Dermatol Ges 11(Suppl6):1-116

2. Hauschild A, Breuninger H, Kaufmann Retal (2013) Brief S2k guidelines - basal cell carcinoma of the skin. J Dtsch Dermatol Ges 11(Suppl3):10-15

3. Jung JW, Overgaard NH, Burke MT et al (2015) Does the nature of residual immune function explain the differential risk of non-melanoma skin cancer development in immunosuppressed organ transplant recipients? Int J Cancer. doi:10.1002/ijc.29450 
4. Askling J, Fored CM, Brandt LL et al (2005) Risks of solid cancers in patients with rheumatoid arthritis and after treatment with tumour necrosis factor antagonists. Ann Rheum Dis 64:1421-1426

5. Greenberg J, Strand V, Keystone E et al (2007) TNF inhibitors (TNF-I) and risk of malignancy in 8,072 RA patients followed over 15,495 patient years (Abstract 282) American College of Rheumatology Annual Meeting

6. Wolfe F, Michaud K (2007) Biologic treatment of rheumatoid arthritis and the risk of malignancy: analyses from a large US observational study. Arthritis Rheum 56:2886-2895

7. Askling J (2009) Anti-TNF therapy and risk of skin cancer, data from the swedish ARTIS registry 1998-2006. Ann Rheum Dis 68(Suppl 3):423

8. MarietteX, Matucci-Cerinic M,Pavelka Ketal (2011) Malignancies associated with tumour necrosis factor inhibitors in registries and prospective observational studies: a systematic review and meta-analysis. Ann Rheum Dis 70:1895-1904

9. Mercer LK, Davies R, Galloway JB et al (2013) Risk of cancer in patients receiving non-biologic diseasemodifying therapy for rheumatoid arthritis compared with the UK general population. Rheumatology 52:91-98

10. Burmester GR, Panaccione R, Gordon KB et al (2013) Adalimumab: long-term safety in 23458 patients from global clinical trials in rheumatoid arthritis, juvenile idiopathic arthritis, ankylosing spondylitis, psoriatic arthritis, psoriasis and Crohn's disease. Ann Rheum Dis 72:517-524

11. Raaschou P, Simard JF, Holmqvist M (2013) Rheumatoid arthritis, anti-tumour necrosis factor therapy, and risk of malignant melanoma: nationwide population based prospective cohort study from Sweden. BMJ346:f1939 doi:10.1136/bmj.f1939

12. Dreyer L, Mellemkjær L, Andersen AR et al (2013) Incidences of overall and site specific cancers in TNFa inhibitor treated patients with rheumatoid arthritis and other arthritides - a follow-up study from the DANBIO registry. Ann Rheum Dis 72:79-82

13. Gross RL, Schwartzman-Morris JS, Krathen M et al (2014) A Comparison of malignancy incidence among psoriatic and rheumatoid arthritis patients in a large US cohort. Arthritis Rheumatol 66:1472-1481

14. Mercer L, Askling J, Raaschou P et al (2014) No increased risk of developing a first invasive melanoma in rheumatoid arthritis patients treated with biologics: results of a collaborative project of 11 european biologics registers. (Abstract 1838) ACR/ARHP Annual Meeting 2014; Boston, MA. Arthritis Rheumatol 66(Supplement):807-808

15. Dixon WG, Watson KD, Lunt Metal (2010) Influence of anti-tumor necrosis factor therapy on cancer incidence in patients with rheumatoid arthritis who have had a prior malignancy: results from the British Society for Rheumatology Biologics Register. Arthritis Care Res (Hoboken) 62:755-763

16. Chakravarty EF, Michaud K, Wolfe F (2005) Skin cancer, rheumatoid arthritis, and tumor necrosis factor inhibitors. JRheumatol 32:2130-2135

17. Leombruno JP, Einarson TR, Keystone EC (2009) The safety of anti-tumour necrosis factor treatments in rheumatoid arthritis: meta and exposure-adjusted pooled analyses of serious adverse events. Ann Rheum Dis 68:1136-1145

18. Mercer LK, Galloway JB, Lunt M et al (2009) The influence of anti-TNF therapy upon incidence of non-melanoma skin cancer (NMSC) in patients with rheumatoid arthritis (RA): results from the
BSR Biologics Register (BSRBR) (abstract). Arthritis Rheum 60(Suppl 10):2062

19. Mercer LK, Green AC, Galloway JB et al (2012) The influence of anti-TNF therapy upon incidence of keratinocyte skin cancer in patients with rheumatoid arthritis: longitudinal results from the British Society for Rheumatology Biologics Register. Ann Rheum Dis 71:869-874

20. Raaschou P, Simard JF, Asker-Hagelberg C et al (2014) Rheumatoid arthritis, anti-tumor necrosis factor therapy, and risk of squamous cell and basa cell skin cancer - a nationwide population based prospective cohort study from Sweden. (Abstract 846) ACR/ARHP Annual Meeting; Boston, MA. Arthritis Rheumatol 66(Supplement):373

21. Scott FI, Mamtani R, Brensinger C et al (2014) Risk of recurrent non-melanoma skin cancer with methotrexate and anti-TNF use in rheumatoid arthritis. (Abstract 1839). ACR/ARHP Annual Meeting 2014; Boston, MA. Arthritis Rheumatol 66(Supplement):808

22. Robinson SN, Zens MS, Perry AE et al (2013) Photosensitizing agents and the risk of nonmelanoma skin cancer: a population-based casecontrol study. J Invest Dermatol 133:1950-1955

23. Gottenberg J-E, Morel J, Ravaud P et al (2015) Tolerance of Rituximab, Abatacept and Tocilizumab in common practice: analysis of the 3 registries of the french society of rheumatology (AIR, ORA and REGATE). EULAR Annual Congress 2015; Rome Italy. Ann Rheum Dis 74(Suppl 2):78

24. Langley RG, Lebwohl M, Krueger GG et al (2015) Long-term efficacy and safety of ustekinumab, with and without dosing adjustment, in patients with moderate-to-severe psoriasis: results from the PHOENIX 2 study through 5 years of follow-up. Br JDermatol 172(5):1371-1383

25. Krathen MS, Gottlieb AB, Mease PJ (2010) Pharmacologic immunomodulation and cutaneous malignancy in rheumatoid arthritis, psoriasis, and psoriatic arthritis. JRheumatol 37(11):2205-2215

26. Van Lümig PP, Menting SP, van den Reek JM et al (2015) An increased risk of non-melanoma skin cancer during TNF-inhibitor treatment in psoriasis patients compared to rheumatoid arthritis patients probably relates to disease-related factors. J Eur Acad Dermatol Venerol 29(4):752-760

27. Reich K, Mrowietz U, Radtke MA et al (2015) Drug safety of systemic treatments for psoriasis: results from The German Psoriasis Registry PsoBest. Arch Dermatol Res. doi:10.1007/s00403-015-1593-8

28. Singh JA, Furst DE, Bharat A et al (2012) Update of the 2008 American College of Rheumatology (ACR) Recommendations for the use of Disease-Modifying Anti-Rheumatic Drugs and Biologics in the treatment of Rheumatoid Arthritis (RA). Arthritis Care Res 64(5):625-639. doi:10.1002/acr.21641

29. Leitlinienentwurf (noch nicht in finaler Version publiziert) für die RA-Therapie, vorgestellt auf dem ACR Congress Boston 2014, http://www. firstreportnow.com/articles/preview-updated2015-draft-guidelines-ra-acr-conferencehighlight. Zugegriffen: 21. Januar 2016

\section{Versorgungsatlas fokussiert erstmals auf seltene Erkran- kungen}

Schätzungen zufolge leiden in Deutschland vier Millionen Menschen an einer seltenen Erkrankung. Nun haben Epidemiologen vom Versorgungsatlas erstmals die Zahl der von einer Orphan Disease betroffenen Menschen in Deutschland erhoben. Dabei mussten sie sich auf jene Erkrankungen beschränken, die mindestens drei von 100.000 Menschen betreffen, eine ICD-10-Kodierung besitzen und keine Infektions- und Tumorkrankheit sind. Insgesamt haben nach diesen Daten niedergelassene Ärzte zwischen 2008 und 2011 pro Jahr im Schnitt über 570.000 Patienten mit einer von 88 seltenen Erkrankungen behandelt. Mit $27 \%$ haben entzündliche Erkrankungen den größten Anteil, gefolgt von genetisch bedingten Erkrankungen (10\%) und Hauterkrankungen (7\%). Bei drei Erkrankungen fanden die Forscher eine Häufigkeit über dem "Schwellenwert" für eine Orphan Disease von 50 pro 100.000 Einwohner. Die Häufigkeiten schwankten zwischen knapp 113 Fällen pro 100.000 bei der Meniére-Krankheit und einem Patienten auf 10 Millionen Einwohner bei der angeborenen Fehlbildung des Zentralnervensystems Kraniorhachischisis.

Ein Beispiel für regionale Unterschiede ist Sarkoidose: In Hessen sind 32 von 100.000 Einwohnern betroffen, während es in Mecklenburg-Vorpommern mit 69 auf 100.000 Einwohner mehr als doppelt so viele sind. Der Versorgungsatlas ist eine Einrichtung des Zentralinstituts für die kassenärztliche Versorgung.

Quelle: Zentralinstitut für die kassenärztliche Versorgung in der

Bundesrepublik Deutschland, www.versorgungsatlas.de 